\title{
The International (Seattle) Criteria
}

\section{CARDIOLOGY / PREPARTICIPATION / SPORTS MEDICINE / TEAM MANAGEMENT}

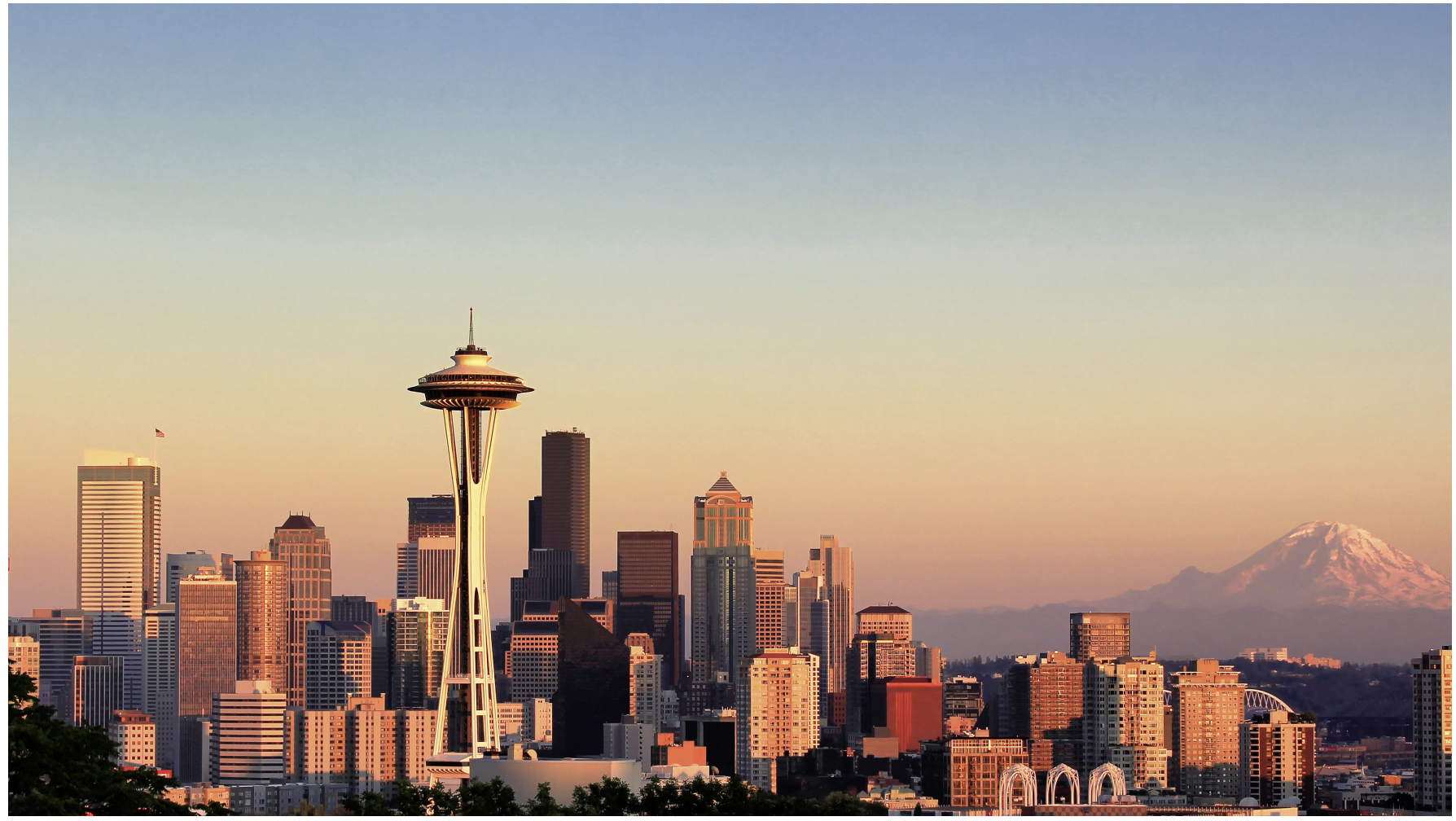

\section{Schmied CM}

Klinik für Kardiologie, Universitäres Herzzentrum Zürich/Schweiz

\section{Abstract}

Through continuous improvement of diagnostic accuracy of ECG criteria for athletes sensitivity as well as specificity have grown so much that foregoing this screening tool is not feasible anymore. The most updated guidelines, the so-called "International (Seattle) Criteria" globally exhibit the most important reference publication, currently. The criteria were created with the purpose that particularly "noncardiologists" should be able to use them before clearly pathological findings lead to further follow-up examinations at a specialist. On the other hand, physiologic ECG findings should not prompt expensive further evaluations, as it used to happen quite frequently. 


\section{Zusammenfassung}

Durch die in den letzten Jahren stetig vorangetriebene Spezifizierung der EKG-Kriterien bei Sportlern konnte eine Sensitivität und auch Spezifität erreicht werden, die ein Übergehen des EKGs im Screening jüngerer Sportler nicht mehr rechtfertigen lässt. Die neusten Richtlinien, die sogenannten «International (Seattle) Criteria» stellen hinsichtlich EKG -

Interpretation bei Sportlern momentan weltweit die Referenzpublikation dar. Sie wurden so konzipiert, dass eine akkurate EKG-Analyse auch durch Nicht-Kardiologen erfolgen kann und soll, bevor bei eindeutig pathologischen Befunden ein Spezialist und weiterführende Untersuchungen hinzugezogen werden. Physiologische Befunde sollen nicht mehr unnötige und kostentreibende Weiterabklärungen nach sich ziehen, wie dies früher oftmals der Fall war.

Die immer wieder aufkommende Debatte über Sinn und Unsinn des kardialen Screenings von Sportlern ist wichtig, doch wird sie auch häufig unseriös und tendenziös geführt [1-4]. Eine der entscheidenden Fragen ist dabei, welche Sportler untersucht werden sollten. Über die Art, wie ein adäquates und evidenzbasiertes Screening durchgeführt wird, besteht, zumindest bei jüngeren Sportlern, weitestgehend Konsens. Diverse Studien konnten dabei neben einer akkuraten Anamnese und fokussierten körperlichen Untersuchung die Wichtigkeit des Ruhe-EKGs zeigen [5,6]. Auf der anderen Seite demonstrierten Baggish et al. eindrücklich, welche Konsequenzen eine Nichtberücksichtigung des EKGs im Screening junger Sportler mit sich bringt: Die Sensitivität von Vorsorgeuntersuchungen, welche einzig aus einer Anamneseerhebung und einer körperlichen Untersuchung bestehen, liegt bei lediglich 45.5\% [7]. Und somit in einem nicht tolerierbar tiefen Bereich. Es stellt sich also die berechtigte Frage nach Gründen, weshalb gerade die grossen amerikanischen Fachgesellschaften die verhältnismässig kostengünstig durchzuführende Untersuchung nicht empfehlen. Mangelnde Verfügbarkeit ist in der heutigen Zeit kaum ein Argument. Das grösste Manko der Untersuchungsmethode ist, oder besser war, sicher ihre ungenügende Spezifität mit dem Potenzial für vor allem viele falsch-positive Befunde [2]. Gerade bei Sportlern sind physiologische EKG-Veränderungen häufig, was immer wieder zu unnötigen und teuren Weiterabklärungen und zur Verunsicherung seitens der Athleten führte. Doch nur weil eine einfache und kosteneffektive Methode ungenügend verstanden wird, rechtfertigt dies selbstverständlich nicht, sie zu ignorieren.

\section{Der erste Seattle Summit}

Es ist erstaunlich aber umso erfreulicher, dass eine amerikanische Gruppe von Sportmedizinern vor einigen Jahren die Initiative ergriff, um der ungenügenden Spezifität des Sportler-EKGs entgegenzuwirken. Jonathan Drezner und seine Gruppe an der University of Washington in Seattle initiierten mit Unterstützung einiger grosser Fachgesellschaften und Sportverbände ein erstes Treffen globaler «OpinionLeader» um die damals gängigen EKG-Richtlinien zu überarbeiten. Die Diskussionen verliefen sehr effizient, ein breiter Konsens konnte rasch erzielt werden, und bald darauf wurden die ersten «Seattle Recommendations» publiziert [8]. Schon damals war es erklärtes Ziel, eindeutig pathologische von für Sportler physiologischen EKG-Veränderungen abzugrenzen. In mehreren Studien konnte in der Folge die Effektivität und deutlich verbesserte Sensitivität und Spezifität der neuen Richtlinien eindrücklich bestätigt werden, und das Konzept wurde in der Folge weltweit zunehmend implementiert. 


\section{Der zweite Seattle Summit 2015 und die aktuellen «International Criteria»}

Aufgrund immer weiterer Daten, welche die EKG-Interpretation eines auch multi-ethnischen Sportlerkollektivs verbesserten, traf die mittlerweile erweiterte Expertengruppe 2015 erneut in Seattle zusammen, um die ursprünglichen Empfehlungen zu aktualisieren. Ergebnis des mehrtägigen Treffens waren die sogennannten «International (Seattle) Criteria» [9]. Aufgrund ihrer ausgezeichneten Sensitivität und Spezifität und des internationalen Expertengremiums konnten die Richtlinien 2017 prominent in mehreren hochklassigen Journals publiziert werden, was einerseits für die Qualität des Dokuments sprach und natürlich auch eine hohe Zahl an interessierten Lesern garantierte [9-11].

Durch die weitere Spezifizierung der Kriterien konnte eine Sensitivität und Spezifität von über 90\% erreicht werden, sodass nun kaum Argumente bestehen bleiben, die einen Verzicht auf das EKG im Screening junger Athleten rechtfertigen würden [12]. Entscheidend ist letztendlich die korrekte Auswahl des zu untersuchenden Athletenkollektivs, doch ist die zuverlässige Detektion von angeborenen zugrunde liegenden Kardiopathien, wie sie zumindest bei Athleten bis zu einem Alter von 30 bis 35 Jahren für den Grossteil der fatalen Ereignisse im Sport verantwortlich sind, nur unter Miteinbezug eines Ruhe-EKGs möglich.

\section{Aufbau und Grundidee der Richtlinien}

Die Vorsorgeuntersuchung von Sportlern sollte durch Fachleute durchgeführt werden, welche regelmässig Sporttreibende untersuchen und mit den besonderen physiologischen und pathophysiologischen Gegebenheiten vertraut sind. Dies bedeutet jedoch keinesfalls, dass dies Kardiologen vorenthalten sein sollte. Um eine breite Abdeckung zuverlässiger Vorsorgeuntersuchungen gewährleisten zu können, ist es zwingend notwendig, dass diese Richtlinien verständlich formuliert und praxisnah implementierbar sind. Dies wird mit den aktuellen Empfehlungen ausgezeichnet umgesetzt. Neben den häufigen physiologischen EKG-Veränderungen, welche keiner weiteren Abklärung bedürfen, werden eindeutig pathologische Veränderungen definiert und mit Empfehlungen zur akkuraten EKG-Analyse und typischen EKGBeispielen untermauert. Fünf Grenzbefunde (EKG-Zeichen für eine Dilatation beider Vorhöfe, elektrische Herzachsenabweichungen und der vollständige Rechtsschenkelblock) sind nur dann abklärungsbedürftig, wenn sie gleichzeitig oder in Kombination mit einem anderen suspekten klinischen Befund auftreten (Abbildung 1).

\section{Physiologische EKG-Veränderungen}

Die zentral-kardiopulmonalen und peripher-muskulären Adaptationen des Sportlers sind eindrücklich und vielseitig [13]. Diese Veränderungen, welche primär durch die Vergrösserung der Herzkammern und den erhöhten Vagotonus bedingt sind, finden auch im Sportler-EKG Ausdruck und sollten nicht zu falschpositiven Interpretationen führen.

Erhöhte QRS-Voltage für links- bzw. rechtsventrikuläre Hypertrophie

Das isolierte Auftreten von positiven Voltage-Kriterien für eine Linksherzhypertrophie korreliert praktisch nie ( $<2 \%)$ mit einer zugrunde liegenden hypertrophen Kardiomyopathie [14], ausser diese sind assoziiert mit T-Negativierungen, ST-Streckensenkungen oder pathologischen Q-Zacken [15]. Isolierte positive Voltage-Kriterien finden sich sehr häufig in Sportler-EKGs und bedürfen keiner weiteren Abklärung. 
Gleiches gilt auch für isoliert auftretende Hypertrophie-Kriterien für eine Rechtsherzhypertrophie [16].

Erhöhte QRS-Voltage für links- bzw. rechtsventrikuläre Hypertrophie

Das isolierte Auftreten von positiven Voltage-Kriterien für eine Linksherzhypertrophie korreliert praktisch nie $(<2 \%)$ mit einer zugrunde liegenden hypertrophen Kardiomyopathie [14], ausser diese sind assoziiert mit T-Negativierungen, ST-Streckensenkungen oder pathologischen Q-Zacken [15]. Isolierte positive Voltage-Kriterien finden sich sehr häufig in Sportler-EKGs und bedürfen keiner weiteren Abklärung. Gleiches gilt auch für isoliert auftretende Hypertrophie-Kriterien für eine Rechtsherzhypertrophie [16].

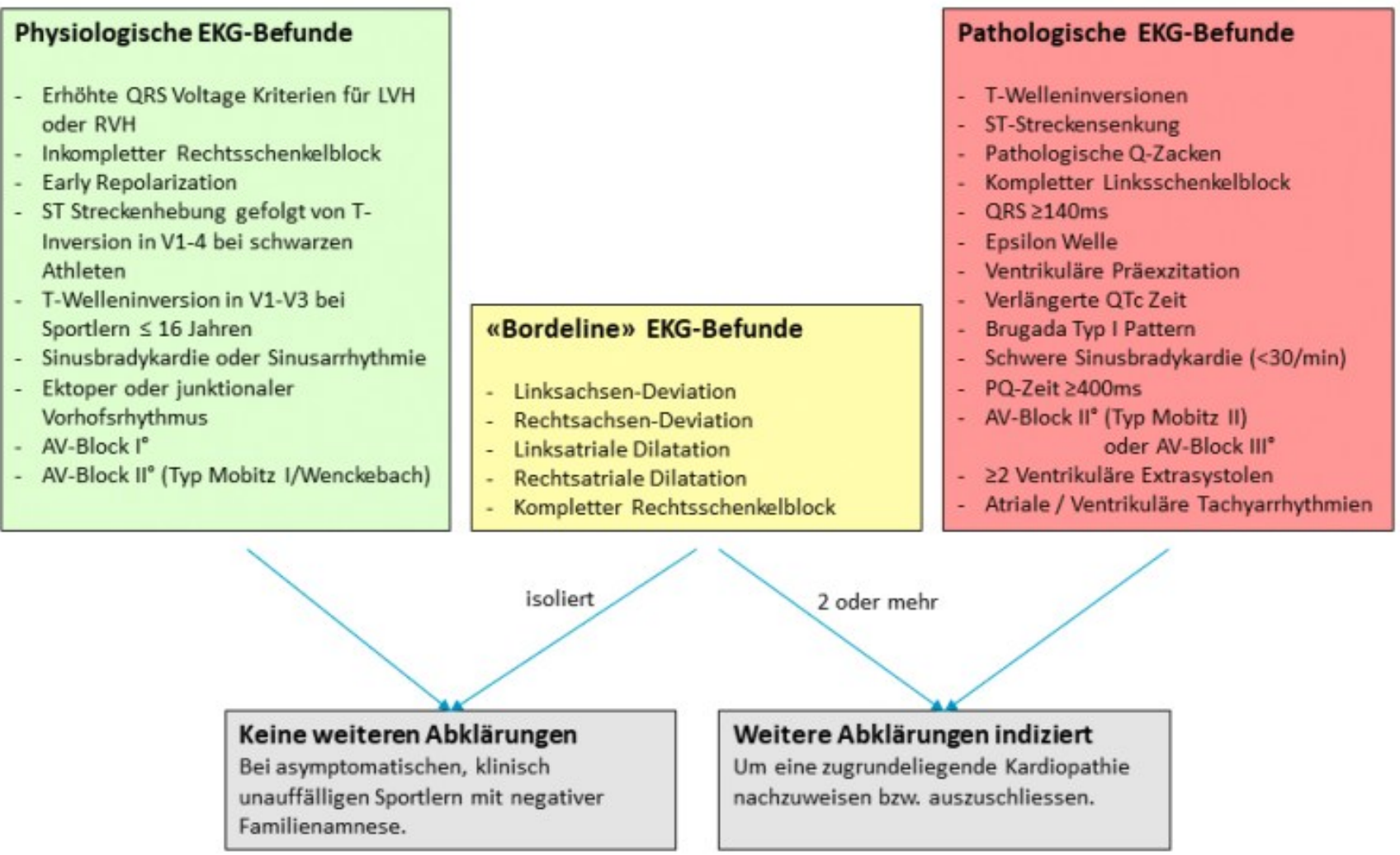

Abb. 1: Die aktuellen «International (Seattle) Criteria in der Übersicht [9].

Early Repolarisation und die ST-Hebung mit nachfolgender T-Welleninversion in V1-V4 bei schwarzen Athleten

Auch wenn in der Literatur oftmals verschiedene Definitionen verwendet werden - die klassische «Early 
Repolarization», definiert als Erhöhung des «J-Punkts» um $\geq 0.1 \mathrm{mV}$, assoziiert mit einem «slurring» oder «notching», ist ein sehr häufiger Befund beim Sportler (2-44\%), vor allem auch bei Sportlern mit ethnisch afro-karibischem Hintergrund [17,18]. Etwa zwei Drittel dieser afro-karibischen Athleten präsentiert sich zudem mit einem klassischen "Early Repolization Pattern”, welches in Verbindung mit einer JPunkterhöhung bzw. einer konvexen ST-Hebung T-Welleninversionen in den anteroseptalen Ableitungen zeigt. Sind diese Veränderungen isoliert und bis maximal Ableitung V1-V4 nachweisbar, wird dieses Muster als benigne angesehen (Abb. 2) [19,20].

Einige wenige Daten bei Überlebenden eines plötzlichen Herzstillstands suggerieren einen Zusammenhang zwischen bestimmten «Patterns» einer Early Repolarization und einem erhöhten Risiko für fatale Arrhythmien. Die Datenlage ist aktuell jedoch noch unzureichend, um derartige Schlüsse zu rechtfertigen [21]. 


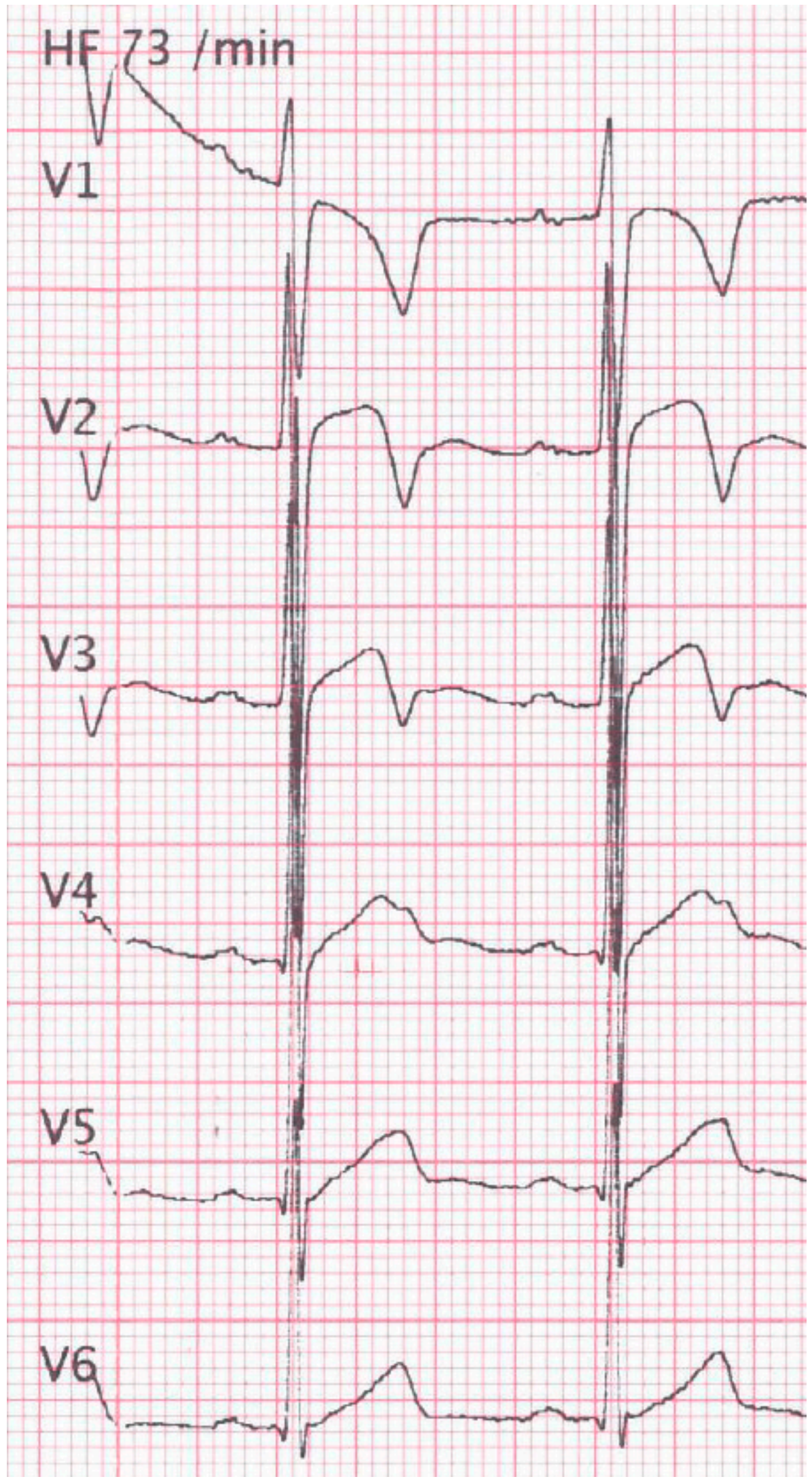

Abb. 2: Typisches (benignes) «Black Athlete’s Early Repolarization Pattern». 
T-Welleninversionen bei Kindern bis zum Alter von 16 Jahren

T-Welleninversionen in den präkordialen Ableitungen V1-V3 werden als physiologischer Befund bei Kindern bis zum Alter von 16 Jahren angesehen, sind Ausdruck einer noch nicht abgeschlossenen Entwicklung und bedürfen keiner weiteren Abklärung [22].

Abbildung 3 gibt einen Überblick über die diagnostischen Kriterien weiterer physiologischer EKGVeränderungen beim Sportler.

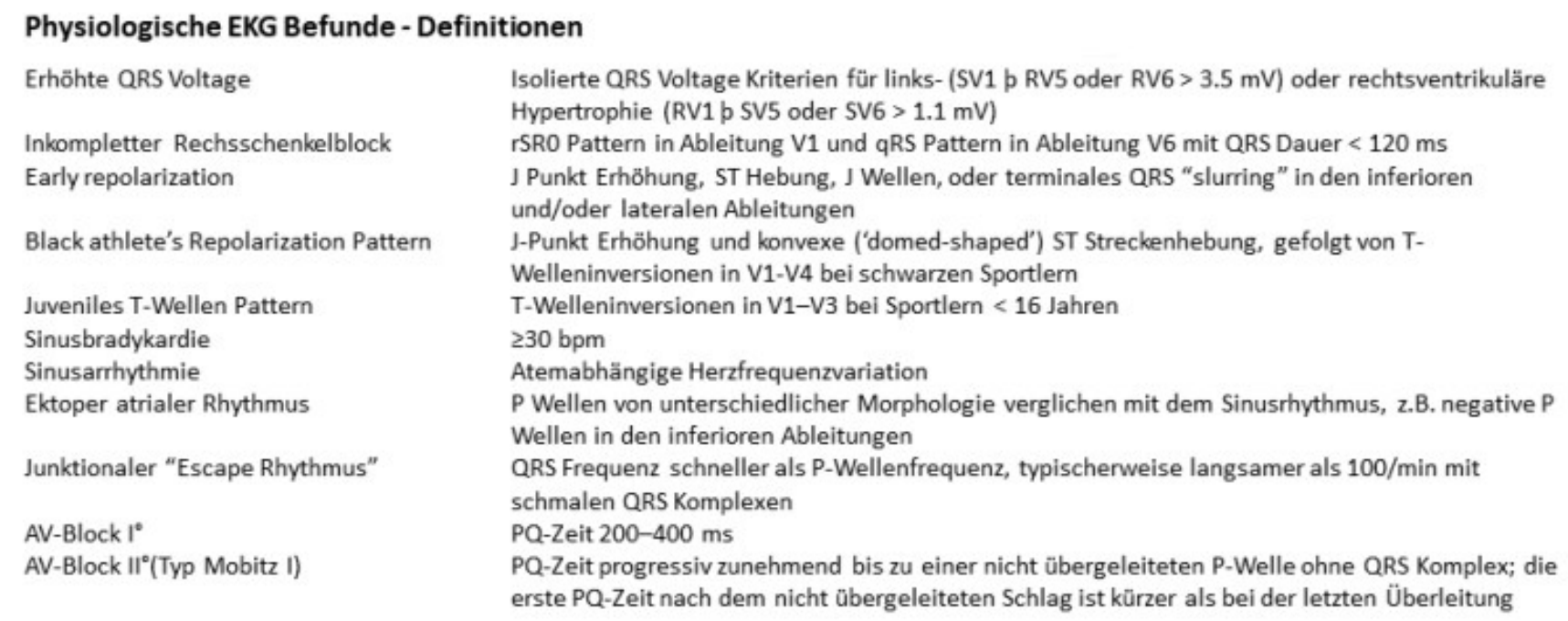

Abb. 3: Überblick über die diagnostischen Kriterien physiologischer EKG-Veränderungen beim Sportler.

\section{Pathologische EKG-Veränderungen}

Pathologische EKG-Veränderung bedürfen weiterer Abklärungen. In der Regel sind diesbezüglich spezialisierte Fachkräfte hinzuzuziehen.

\section{T-Welleninversionen}

T-Welleninversionen (TWI) von $\geq 1 \mathrm{~mm}$ Tiefe, in zwei oder mehr kontinuierlichen Ableitungen (mit Ausnahme der Ableitungen III; aVR und V1), sind pathologisch und sollten zwingend weiter abgeklärt werden. Die Korrelation von TWI und verschiedenen zugrunde liegenden Kardiopathien ist klar erwiesen $[23,24]$. 
Mit Ausnahme von benignen anterioren TWI, wie in V1-V3 bei Kindern $\leq 16$ Jahren oder beim klassischen «Early Repolarization Pattern» von schwarzen Athleten sind diese Veränderungen hoch suspekt und abklärungsbedürftig. Debattiert wird einzig über die zulässige Ausdehnung der T-Negativierungen über Vorderwand [25]: Eine grosse Follow-up-Studie bei über 2500 Athleten konnte zeigen, dass TWI über den Ableitungen V1-V2 relativ häufig sind und dabei, auch im Verlauf, keine zugrunde liegenden Pathologien ergaben [26]. Bei Sportlern mit tiefem kardialem Risiko bergen diese spezifischen Veränderungen also keinen Grund zur Besorgnis, regelmässige Verlaufskontrollen sind aber sicher angezeigt. TWI, welche sich jedoch über die Ableitungen V1-V2 hinaus ausbreiten, sind abklärungsbedürftig, wobei gerade anteroseptale TWI den Verdacht auf eine rechtsventrikuläre Pathologie (Arrhythmogene Rechtsventrikuläre Kardiomyopathie/ARVC) aufkommen lassen [27]. Insbesondere, wenn die TWI in Assoziation mit einer Delta-Welle, einem verlängerten S-Zacken "upstroke», einer peripheren Niedervoltage und Extrasystolen mit Linksschenkelblock-Morphologie zu finden sind [24].

\section{Laterale und inferolaterale TWI}

Im Falle von TWI über den lateralen und inferolateralen Ableitungen besteht immer ein hochgradiger Verdacht auf eine zugrunde liegende Kardiomyopathie [20,28]. Weiterführende Abklärungen sollten neben einer Bildgebung mittels Echokardiographie (und gegebenenfalls MRI) auch ein Belastungs- und Langzeit-EKG umfassen.

\section{Inferiore TWI}

Die Signifikanz von begrenzt inferior auftretenden TWI ist nicht sicher. Dennoch können diese Repolarisationsstörungen keinem physiologischen Korrelat zugeordnet werden. Und so gilt ebenfalls die Empfehlung für eine Weiterabklärung mit zumindest einer Echokardiographie.

\section{ST-Streckensenkungen}

ST-Streckensenkungen ( $\geq 0.05 \mathrm{mV})$, im Verhältnis zur PQ-Strecke als Referenz, in zwei oder mehr konsekutiven Ableitungen sind sehr spezifisch für eine korrelierende Kardiomyopathie (in 60-70\% Hypertrophe Kardiomyopathie) und sollten eingehende Untersuchungen hinsichtlich dieser Erkrankungen nach sich ziehen [29].

\section{Pathologische Q-Zacken}

Verschiedene Kardiopathien, wie etwa Kardiomyopathien, Myokarditis, akzessorische Leitungsbahnen oder eine Koronare Herzkrankheit mit transmuralem Infarkt, können in pathologischen Q-Zacken ihren Ausdruck finden [30]. Wichtig ist dabei eine akkurate Definition pathologischer Q-Zacken, da viele ältere Richtlinien, welche sich lediglich an der Q-Zackentiefe orientierten, zu einer grossen Anzahl falschpositiver Befunde führten. Es wird deshalb empfohlen, die Q-Zacken-Amplitude in Relation zur R-Zacke zu setzen und zudem die Zackenweite zu beurteilen [31]. Eine Q/R Ratio von $\geq 0.25$ bzw. eine Q-Zackendauer 
von $\geq 40 \mathrm{~ms}$ in mindestens zwei konsekutiven Ableitungen (mit der Ausnahme von III und aVR) wird deshalb als klar pathologisch beurteilt. Isolierte Q-Zacken in V1 und V2 sollten eine Wiederholung der Untersuchung nach sich ziehen, da der Verdacht auf eine Elektrodenfehlpositionierung besteht.

\section{Kompletter Linksschenkelblock und unspezifische QRS-Verbreiterung $\geq 140 \mathrm{~ms}$}

Ein kompletter Linksschenkelblock ist ein extrem seltener Befund bei Athleten $(<0.1 \%)$ und suggeriert praktisch immer eine zugrunde liegende Erkrankung (z.B. Kardiomyopathie oder KHK) oder zumindest eine ungünstige Prognose [32].

Ebenso stellt sich ein unspezifischer Schenkelblock mit deutlich prolongierter QRS-Zeit ( $\geq 140 \mathrm{~ms})$ als klar pathologischer EKG-Befund dar [33].

\section{Ventrikuläre Präexzitation}

Ventrikuläre Präexzitation tritt immer dann auf, wenn die AV-Überleitung über zusätzlich akzessorische Bahnen erfolgt. Sie präsentiert sich im EKG typischerweise mit einer Verkürzung der PQ-Zeit und einer Verbreiterung des QRS-Komplexes (im Sinne eines Fusionskomplexes). Ein typisches Pattern stellt das sogenannte «Wolff-Parkinson-White»-Muster, mit verkürzter PQ-Zeit ( $<120 \mathrm{~ms}$ ) und einer Delta-Welle im ansteigenden Teil der R-Zacke, dar [34]. Das WPW-Pattern zeigt sich in etwa einem von 250 Athleten [5,35]. Das Vorliegen einer akzessorischen AV-Überleitung kann durch die schnelle Überleitung von supraventrikulären Rhythmusstörungen gefährlich werden. Insbesondere beim Vorhofflimmern. Bei asymptomatischen und anamnestisch unauffälligen Sportlern stellt eine isolierte PQStreckenverkürzung, ohne zusätzliche Verbreiterung des QRS-Komplexes, keine Indikation für weitere Abklärungen dar. Beim Vorliegen eines WPW-Patterns sollte nach einer initialen nicht-invasiven Beurteilung mittels Ergometrie (zur Abschätzung der Leitungsgeschwindigkeit der akzessorischen Bahn) die Indikation zur elektrophysiologischen Abklärung (und ggf. gleichzeitigen Therapie) grosszügig gestellt werden [36].

Ausserdem sollte mittels Echokardiographie eine allfällig assoziierte strukturelle Herzkrankheit gesucht werden (z.B. Kardiomyopathie, Ebstein-Anomalie).

\section{Verlängerte QTc-Zeit}

Das kongenitale Long-QT-Syndrom (LQTS) ist ein potenziell lebensgefährliches, genetisch determiniertes ventrikuläres Arrhythmiesyndrom mit dem klassischen EKG-Befund einer verlängerten QTc-Zeit. Das LQTS tritt bei etwa einem von 2000 Individuen auf, wird aber unterschätzt, da ein Syndrom auch bei, zumindest vorübergehend, normaler QT-Zeit vorliegen kann (sog. concealed LQTS) [37]. Das LQTS ist in bis zu 25-40\% Autopsie negativer plötzlicher Herztode für die fatalen Ereignisse verantwortlich [38]. Zur Diagnosestellung ist die korrekte Messung der relevanten QT-Zeit essenziell und die Zuverlässigkeit der Messung kann mittels sechs einfacher Regeln optimiert werden [39]:

- Zur Frequenzkorrektur der QT-Zeit (QTc) sollte die "Bazett”-Formel verwendet werden (QTc = QT/RR- 
Intervall in Sekunden) [40].

- Die Bazett-Formel unterschätzt die QTc bei Herzfrequenzen <50/min und überschätzt die QTc bei Herzfrequenzen $>90 / \mathrm{min}$. Dies sollte bei der Durchführung des EKGs bedacht werden. Bei sehr tiefen Frequenzen muss die Herzfrequenz mittels einer kurzen körperlichen Belastung angehoben werden, um die QTc akkurat berechnen zu können.

- Falls eine Sinusarrhythmie und somit eine Variabilität der Herzfrequenz vorliegt, sollte ein Durchschnittswert für die QT-Zeit und das RR-Intervall verwendet werden.

- Die Ableitungen II und V5 gewähren üblicherweise die optimalsten Bedingungen, um die QT-Zeit zu messen.

- U-Wellen, welche sich typischerweise über den Brustwandableitungen finden, dürfen nicht in die QTMessung/Berechnung einbezogen werden («teach the tangent - avoid the tail») [39].

- Gewisse morphologische Kennzeichen der T-Welle können zusätzliche Hinweise für das Vorliegen eines LQTS geben [41]. Zum Beispiel ein «notch» der T-Welle in den lateralen präkardialen Ableitungen beim LQTS 2.

Um die Zuverlässigkeit der vom Computer gemessenen Werte zu beurteilen, bietet sich an, eine stichprobenartige Selbstmessung in den Ableitungen II und V5 durchzuführen. Liegt die eigene Messung innerhalb von 10ms der Computermessung, können die Computerwerte übernommen werden. Im Rahmen der Seattle-Konferenzen wurden die bis dahin relativ tiefen «cut-offs» zur Definition einer QTc-Verlängerung angepasst. So wird nun neu empfohlen, dass eine Weiterabklärung erfolgen soll, wenn die QTc $\geq 470 \mathrm{~ms}$ bei Männern und $\geq 480 \mathrm{~ms}$ bei Frauen liegt. Mit diesen höheren Grenzwerten wird die Balance zwischen tatsächlich pathologischen Messwerten und dem positiv prädiktiven Wert für ein LQTS gewährleistet [42].

Zur korrekten Diagnosestellung aber auch Risikobeurteilung gehört es nicht nur, die oben beschriebenen «cut-offs» zu überschreiten. Vielmehr sollte eine individuelle Risikobeurteilung erfolgen. Eine positive persönliche Anamnese hinsichtlich Synkopen und Krampfanfällen und eine positive Familienanamnese für Synkopen, Krampfanfälle oder unklare Todesfälle ist alarmierend, und der Sportler sollte zwingend fachärztlich abgeklärt werden. Wenn die Anamnese andererseits unauffällig ist, wird empfohlen, wiederholte EKGs an verschiedenen Tagen durchzuführen. Zeigen sich zwischenzeitlich auch normalisierte QTc-Messungen, sind keine weiteren Abklärungen angezeigt.

Sollte die QTc persistierend relevant verlängert bleiben, wird ein Familienscreening und eine weiterführende rhythmologische Abklärung empfohlen.

Bei Vorliegen einer QTc von $\geq 500 \mathrm{~ms}$ sollte der Sportler auf jeden Fall fachärztlich abgeklärt werden, es sei denn, eine eindeutig assoziierte, reversible Ursache (z.B. eine Elektrolytstörung) liegt vor [43]. Zu empfehlen ist ausserdem der Einbezug des «Schwartz-Moss»-Schemas zur prognostischen Beurteilung eines LQTS [44].

\section{Brugada-Typ-I-Muster}

Das Brugada-Syndrom (BrS) ist eine hereditäre Ionenkanalerkrankung, die, vor allem in Phasen von erhöhtem Vagotonus, zu fatalen ventrikulären Arrhythmien führen kann. Die EKG-Blickdiagnose wird ermöglicht durch ein typisches EKG-Muster, primär ersichtlich in den Ableitungen V1-V3 mit einem rSr'Komplex, einer ST-Hebung $\geq 2 \mathrm{~mm}$ und einer terminalen T-Welleninversion (Abb. 4) [45]. Bei unsicherem Befund kann ein Umplatzieren der Elektroden V1 und V2 manchmal ein Brugada-EKG 
demaskieren (Umsetzen der Elektroden vom 4. in den 3. oder 2. Intercostalraum), und es erscheint das typische rSr'-Pattern.

Der Sportler mit typischem Typ-1-Brugada-EKG sollte, unabhängig von Symptomen und Anamnese, immer rhythmologisch abgeklärt werden.

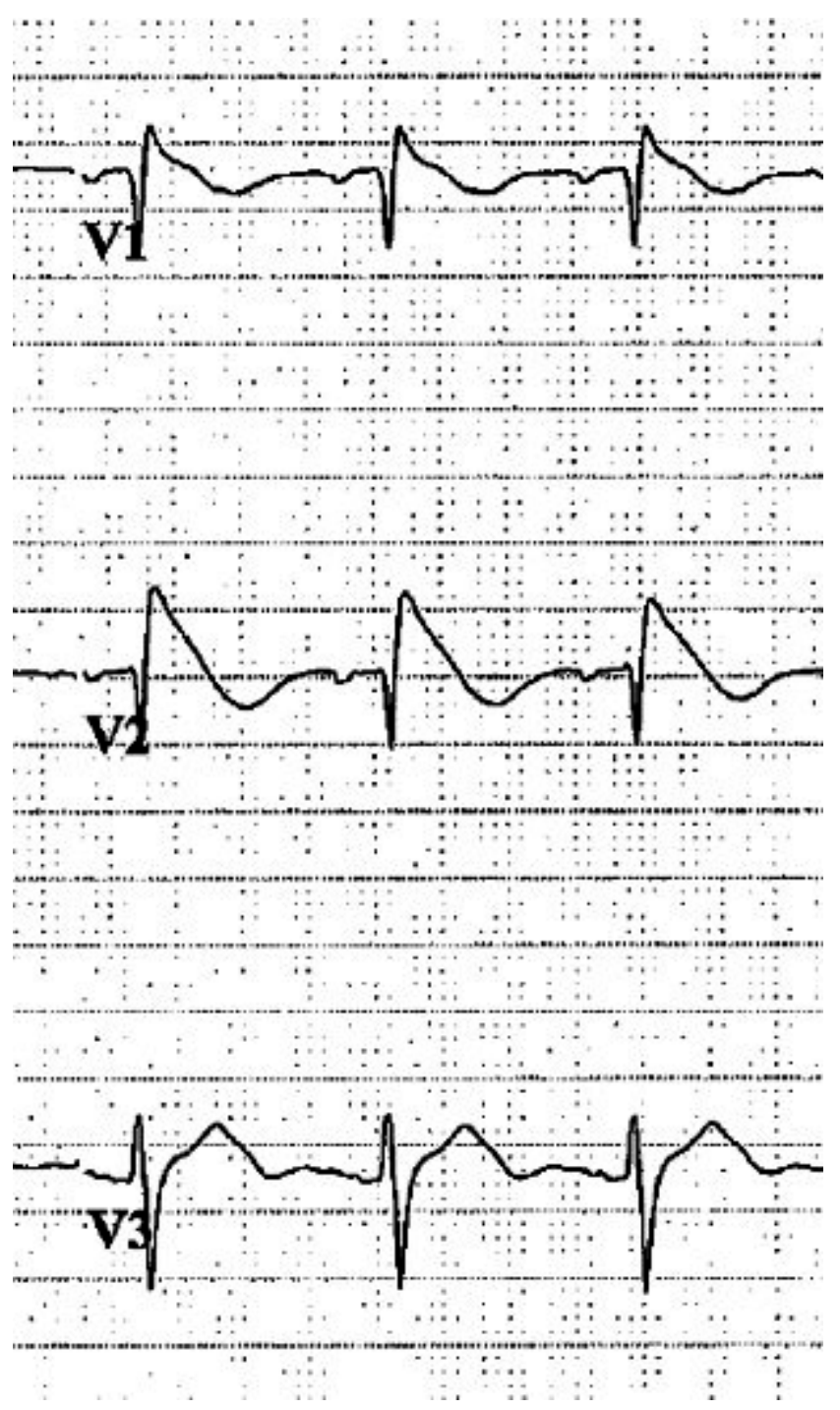

Alblo. 4: Typisches Brugada-Typ-I-Pattern mit rSr’Komplex.

Schwere Sinusbradykardie und höhergradiger AV-Block

Da bei gut trainierten Sportlern eine Sinusbradykardie und ein tiefgradiger AV-Block aufgrund des erhöhten Vagotonus' sehr häufig auftritt, werden nur schwere Sinusbradykardien $(\leq 30 / \mathrm{min})$ und höhergradige AV-Blockbilder (PQ-Zeit $\geq 400 \mathrm{~ms}$, AV-Block Grad 2 (Typ Mobitz II) und AV-Block Grad III) weiter abgeklärt.

Sollten sich die prolongierten Strecken unter Belastung (z.B. während einer Ergometrie) nicht 
normalisieren oder zumindest verkürzen, ist eine rhythmologische Weiterabklärung indiziert. Wie selbstverständlich auch bei symptomatischen Athleten.

\section{Vermehrte ventrikuläre Extrasystolen}

Multiple ventrikuläre Extrasystolen (VES) ( $\geq 2$ pro 10 Sekunden EKG-Aufzeichnung) sind insgesamt selten und finden sich in weniger als bei 1\% der Athleten [5,35]. Obwohl ventrikuläre Extrasystolen meist benigne sind, können sie gleichwohl Ausdruck einer zugrunde liegenden Herzerkrankung sein [46]. VES aus dem rechtsventrikulären Ausflusstrakt (LSB-pattern mit inferiorer Achse) werden als gutartig erachtet, wenn sich das übrige EKG normal präsentiert. Dennoch können ventrikuläre Extrasystolen derselben Morphologie auch bei relevanten Erkrankungen, wie etwa einer ARVC, auftreten, weshalb der Konsens gefunden wurde, dass $\geq 2$ VES pro 10 Sekunden EKG-Aufzeichnung weitere Abklärungen nach sich ziehen sollten. Finden sich Normalbefunde in der Echokardiographie (evtl. MRI), im Holter-EKG ( $<2000 \mathrm{VES} / 24 \mathrm{~h}$ ) und in der Ergometrie (Abnahme der VES unter Belastung) sind beim asymptomatischen Athleten keine weiteren Abklärungen indiziert. Eine rezente Studie konnte eine Korrelation zwischen dem Auftreten von mindestens 2000 VES pro 24 Stunden und einer zugrunde liegenden Kardiopathie in 30\% der Athleten zeigen [46], weshalb bei Sportlern mit dieser Konstellation, neben einer Echokardiographie, auch ein Herz-MRI durchgeführt werden sollte (u.a. auch mit der Frage nach Fibrose) [47].

\section{Atriale und ventrikuläre Tachyarrhythmien}

Selbstverständlich benötigen Athleten mit supraventrikulären oder ventrikulären Tachyarrhythmien weitere Abklärungen. Diese Rhythmusstörungen führen in den meisten Fällen zu einer deutlichen Symptomatik und bergen das Potenzial für relevante Komplikationen.

Abbildung 5 gibt einen Überblick über die diagnostischen Kriterien von pathologischen EKGVeränderungen beim Sportler.

\section{Grenzbefunde}

Elektrokardiographische Grenzbefunde sind nur dann abklärungsbedürftig, wenn sie gleichzeitig oder in Kombination mit einem anderen suspekten klinischen Befund auftreten.

\section{Überdrehter Lagetyp und Vorhofsdilatation}

Die Erkenntnis, dass überdrehte elektrische Herzachsen und Voltage-Kriterien für eine Vergrösserung der Vorhöfe zwar häufige Befunde bei Sportlern darstellen (> 40\%) aber nur extrem selten mit zugrunde liegenden Pathologien korrelieren, führte im Wesentlichen dazu, dass die Spezifität der Seattle-Kriterien derart verbessert werden konnte [48].

Diese Veränderungen sind nur in Kombination als pathologisch anzusehen. 
Die Datenlage, welche die Dignität des kompletten Rechtsschenkelblocks (RSB) bei Sportlern beurteilen lässt, ist weniger eindeutig. Basierend auf grösseren Studien und Datensätzen in der Normalbevölkerung wird momentan von einer Prävalenz des RSB um 2.5\% ausgegangen. Eine Follow-up- Studie konnte dabei keinen Zusammenhang mit zugrunde liegenden strukturellen Kardiopathien feststellen, weshalb aktuell eine Weiterabklärung nur dann empfohlen wird, wenn der Befund in Assoziation mit anderen EKGBefunden oder klinischen Auffälligkeiten auftritt (49). Abbildung 6 gibt einen Überblick über die diagnostischen Kriterien von Grenzbefunden des Sportler-EKGs.

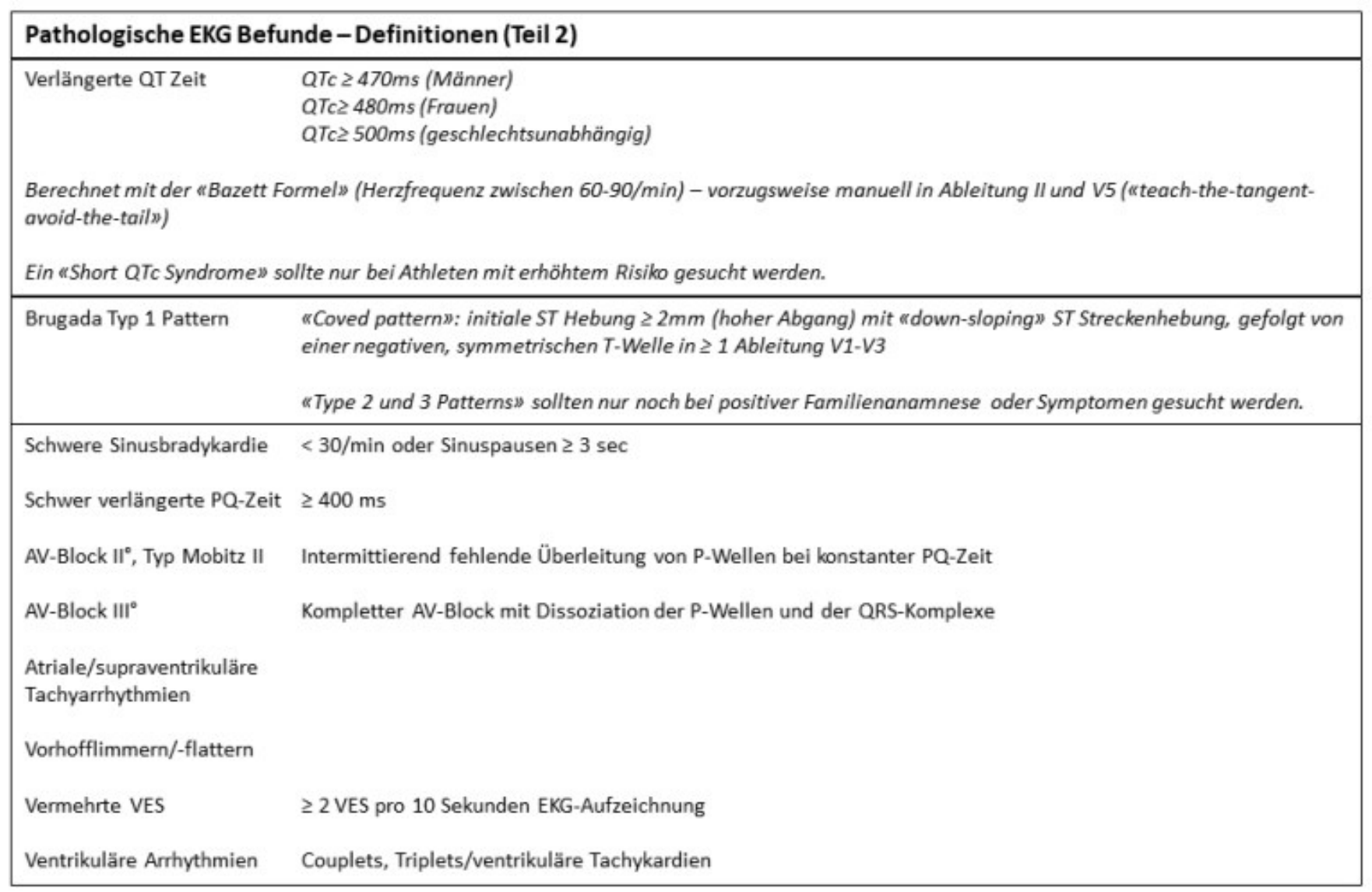

\section{Abb. 5: Überblick über die diagnostischen Kriterien pathologischer EKG-Veränderungen beim Sportler.}

\section{Grenzwertige EKG Befunde - Definitionen}

Linksachsendeviation Rechtsachsendeviation Linksatriale Dilatation Rechtsatriale Dilatation Kompletter Rechtsschenkelblock $-30^{\circ}$ bis $-90^{\circ}$

$>120^{\circ}$

P Welle $>120 \mathrm{~ms}$ in Ableitung I oder II mit negativem P Wellen Anteil $\geq 40 \mathrm{~ms}$ in Ableitung V1

$P$ Wellen Amplitude $\geq 2.5 \mathrm{~mm}$ in II, III oder aVF

rSR' pattern in Ableitung V1 und S Zacke breiter als R Zacke in Ableitung V6 und QRS $\geq 120 \mathrm{~ms}$ 


\begin{tabular}{|c|c|}
\hline Pathologische EKG Befun & nde-Definitionen (Teil 1) \\
\hline T Wellen Inversionen mind. 1 & $1 \mathrm{~mm}$ tief in $\geq 2$ kontinuierlichen Ableitungen (exkl aVR, III and V1) \\
\hline - Anterior & $\begin{array}{l}\text { V3-V4 } \\
\text { (ausser schwarze Athleten mit J-point Erhöhung und konvexer ST Streckenhebung gefolgt von T-Wellen } \\
\text { Inversion V2-V4; und Sportler < } 16 \text { Jahren mit T-Wellen Inversion V1-V3; und biphasische T Welle in V3) }\end{array}$ \\
\hline Lateral & $\begin{array}{l}\text { I, aVL, V5 und/oder V6 (nur eine Ableitung nōtig in V5/V6) } \\
\text { eingehende Abklärung indiziert! }\end{array}$ \\
\hline - Inferolateral & II und aVF, V5-V6, I und aVL \\
\hline & eingehende Abklärung indiziert! \\
\hline - Inferior & II und aVF isoliert \\
\hline & kein ausgesprochen starker Prädiktor für zugrundeliegende Kardiopathie \\
\hline ST Strecken Senkung & $\geq 0.5 \mathrm{~mm}$ in $\geq 2$ kontinuierlichen Ableitungen (im Verhältnis zur isoelektrischen PQ Strecke) \\
\hline Pathologische Q Zacken & $Q / R$ ratio $\geq 0.25$ oder $Q$ Zacken Dauer $\geq 40 \mathrm{~ms}$ in $\geq 2$ Ableitungen (ausser III, $a V R$ ) \\
\hline $\begin{array}{l}\text { Kompletter } \\
\text { Linksschenkelblock }\end{array}$ & $\begin{array}{l}\text { QRS } \geq 120 \mathrm{~ms} \text { Mehrheitlich negativer QRS Komplex in Ableitung V1 ("QS oder rS») und aufrechte "notched or } \\
\text { slurred» R Zacke in Ableitung I und V6 }\end{array}$ \\
\hline $\begin{array}{l}\text { Hochgradiger, } \\
\text { unspezifischer Schenkelblock }\end{array}$ & QRS $\geq 140 \mathrm{~ms}$ \\
\hline Epsilon wave & $\begin{array}{l}\text { Diskretes positives Potenzial bzw. "notch" zwischen dem Ende des QRS Komplexes und dem Beginn der T Welle } \\
\text { in den Ableitungen V1-V3 }\end{array}$ \\
\hline Ventrikuläre Prä-Exzitation & PQ Intervall < 120ms mit Delta Welle («slurred» upstroke) und verbreiterter QRS Komplex ( $\geq 120 \mathrm{~ms}$ ) \\
\hline
\end{tabular}

\section{Konklusion und Ausblick}

Durch die in den letzten Jahren stetig vorangetriebene Spezifizierung der EKG-Kriterien bei Sportlern konnte eine Sensitivität und auch Spezifität erreicht werden, die ein Übergehen des EKGs im Screening jüngerer Sportler heutzutage nicht mehr rechtfertigen lässt. Die neusten Richtlinien, die sogenannten «International (Seattle) Criteria» stellen momentan weltweit die Referenzpublikation dar. Sie wurden so konzipiert, dass eine akkurate EKG-Analyse auch durch Nicht-Kardiologen erfolgen kann und soll, bevor bei eindeutig pathologischen Befunden ein Spezialist und weiterführende Untersuchungen hinzugezogen werden.

Wichtig zu erwähnen ist die Tatsache, dass mittels EKG-integriertem Basisscreening nur die zugrunde liegenden, meist angeborenen Erkrankungen bei jüngeren Athleten adäquat detektiert werden können. Bei älteren Sportlern, ab einem Alter von 30 bis 35 Jahren ist in über 80\% eine koronare Plaqueruptur (bzw. ein Myokardinfarkt) für plötzliche, sportassoziierte Herztodesfälle verantwortlich. Und diese können selbstverständlich mit diesem Standard-Basisscreening nicht genügend detektiert werden [38]. Alarmierend sind in diesem Zusammenhang auch Daten, die bereits bei jüngeren Athleten in 
zunehmendem Masse eine koronare Herzkrankheit als Ursache für fatale kardiale Ereignisse zeigen [50].

\section{Corresponding author}

PD Dr. med. Christian Marc Schmied

Leitender Arzt

Klinik für Kardiologie

Universitäres Herzzentrum

Rämistrasse 100

CH-8091 Zürich

Tel. +41442553478

Email: christian.schmied@usz.ch

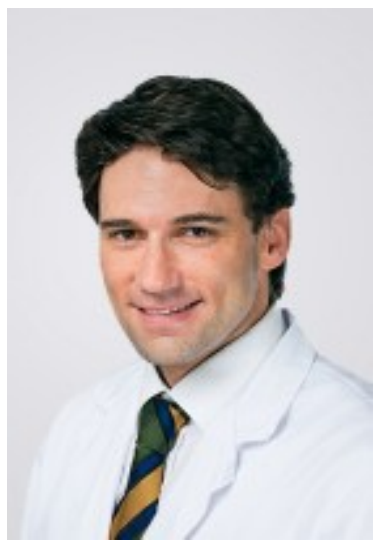

\section{References}

1. Corrado D, Pelliccia A, Bjornstad HH, Vanhees L, Biffi A, Borjesson M, et al. Cardiovascular preparticipation screening of young competitive athletes for prevention of sudden death: proposal for a common European protocol. Consensus Statement of the Study Group of Sport Cardiology of the Working Group of Cardiac Rehabilitation and Exercise Physiology and the Working Group of Myocardial and Pericardial Diseases of the European Society of Cardiology. Eur Heart J 2005;26:516-524.

2. Chaitman BR. An electrocardiogram should not be included in routine preparticipation screening of young athletes. Circulation. 2007 Nov 27;116(22):2610-4; discussion 2615.

3. Schmied C, Notz S. Cardiac pre-competiton screening in Swiss athletes. Current situation in competitive athletes and short-time assessment of an exemplary local screening program. Swiss Med Wkly. 2012 May 31;142:w13575.

4. Van Brabandt H, Desomer A, Gerkens S, Neyt M. Harms and benefits of screening young people to prevent sudden cardiac death. BMJ. 2016 Apr 20;353:i1156.

5. Pelliccia A, Culasso F, Di Paolo FM, Accettura D, Cantore R, Castagna W, et al. Prevalence of abnormal electrocardiograms in a large, unselected population undergoing pre-participation cardiovascular screening. Eur Heart J 2007;28:2006-2010.

6. Corrado D, Basso C, Pavei A, Michieli P, Schiavon M, Thiene G. Trends in sudden cardiovascular death in young competitive athletes after implementation of a preparticipation screening program. JAMA. 2006 Oct 4;296(13):1593-601.

7. Baggish AL, Hutter AM Jr, Wang F, Yared K, Weiner RB, Kupperman E, et al. Cardiovascular screening in college athletes with and without electrocardiography: A cross-sectional study. Ann Intern Med. 2010 Mar 2;152(5):269-75.

8. Drezner JA, Ackerman MJ, Anderson J, Ashley E, Asplund CA, Baggish AL, et al. Electrocardiographic interpretation in athletes: the "Seattle criteria" . Br J Sports Med 2013;47:122-124.

9. Sharma S, Drezner JA, Baggish A, Papadakis M, Wilson MG, Prutkin JM, et al. International recommendations for electrocardiographic interpretation in athletes. Eur Heart J. 2018 Apr 21;39(16):1466-1480.

10. Sharma S, Drezner JA, Baggish A, Papadakis M, Wilson MG, Prutkin JM, et al. International Recommendations for Electrocardiographic Interpretation in Athletes. J Am Coll Cardiol. 2017 Feb 
28;69(8):1057-1075.

11. Drezner JA, Sharma S, Baggish A, Papadakis M, Wilson MG, Prutkin JM, et al. International criteria for electrocardiographic interpretation in athletes: Consensus statement. Br J Sports Med. 2017 May;51(9):704-731.

12. Dhutia H, Malhotra A, Finocchiaro G, Merghani A, Papadakis M, Naci H, et al. Impact of the International Recommendations for Electrocardiographic Interpretation on Cardiovascular Screening in Young Athletes. Journal of the American College of Cardiology Aug 2017, 70 (6) 805-807.

13. Baggish AL, Wood MJ. Athlete's heart and cardiovascular care of the athlete: scientific and clinical update. Circulation. 2011 Jun 14;123(23): 2723-35.

14. Calore C, Melacini P, Pelliccia A, Cianfrocca C, Schiavon M, Di Paolo FM, et al. Prevalence and clinical meaning of isolated increase of QRS voltages in hypertrophic cardiomyopathy versus athlete's heart: relevance to athletic screening. Int J Cardiol 2013;168:4494-4497.

15. Lakdawala NK, Thune JJ, Maron BJ, Cirino AL, Havndrup O, Bundgaard H, et al. Electrocardiographic features of sarcomere mutation carriers with and without clinically overt hypertrophic cardiomyopathy. Am J Cardiol 2011;108:1606-1613.

16. Zaidi A, Ghani S, Sheikh N, Gati S, Bastiaenen R, Madden B, et al. Clinical significance of electrocardiographic right ventricular hypertrophy in athletes: comparison with arrhythmogenic right ventricular cardiomyopathy and pulmonary hypertension. Eur Heart J 2013; 34:3649-3656.

17. Uberoi A, Jain NA, Perez M, Weinkopff A, Ashley E, Hadley D, et al. Early repolarization in an ambulatory clinical population. Circulation 2011;124:2208-2214.

18. Noseworthy PA, Weiner R, Kim J, Keelara V, Wang F, Berkstresser B, et al. Early repolarization pattern in competitive athletes: clinical correlates and the effects of exercise training. Circ Arrhythm Electrophysiol 2011;4:432-440.

19. Di Paolo FM, Schmied C, Zerguini YA, Junge A, Quattrini F, Culasso F, et al. The athlete's heart in adolescent Africans: an electrocardiographic and echocardiographic study. J Am Coll Cardiol 2012; 59:1029-1036.

20. Papadakis M, Carre F, Kervio G, Rawlins J, Panoulas VF, Chandra N, et al. The prevalence, distribution, and clinical outcomes of electrocardiographic repolarization patterns in male athletes of African/Afro-Caribbean origin. Eur Heart J 2011;32:2304-2313.

21. Haissaguerre M, Derval N, Sacher F, Jesel L, Deisenhofer I, de Roy L, et al. Sudden cardiac arrest associated with early repolarization. N Engl J Med 2008;358:2016-2023.

22. Migliore F, Zorzi A, Michieli P, Perazzolo Marra M, Siciliano M, et al. Prevalence of cardiomyopathy in Italian asymptomatic children with electrocardiographic Twave inversion at preparticipation screening. Circulation 2012;125:529-538.

23. Chen X, Zhao T, Lu M, Yin G, Xiangli W, Jiang S, et al. The relationship between electrocardiographic changes and CMR features in asymptomatic or mildly symptomatic patients with hypertrophic cardiomyopathy. Int J Cardiovasc Imaging 2014;30 Suppl 1:55-63.

24. Marcus FI, McKenna WJ, Sherrill D, Basso C, Bauce B, Bluemke DA, et al. Diagnosis of arrhythmogenic right ventricular cardiomyopathy/dysplasia: proposed modification of the task force criteria. Circulation 2010;121:1533-1541.

25. Drezner JA, Ashley E, Baggish AL, Borjesson M, Corrado D, Owens DS, et al. Abnormal electrocardiographic findings in athletes: recognising changes suggestive of cardiomyopathy. $\mathrm{Br} \mathrm{J}$ Sports Med 2013;47:137-152.

26. Malhotra A, Dhutia H, Gati S, Yeo T-J, Dores H, Bastiaenen R, et al. Prevalence and significance of anterior T wave inversion in young white athletes and non athletes. JACC 2016;69:1-9.

27. Calore C, Zorzi A, Sheikh N, Nese A, Facci M, Malhotra A,et al. Electrocardiographic anterior T-wave inversion in athletes of different ethnicities: differential diagnosis between athlete's heart and cardiomyopathy. Eur Heart J 2015;37:2515-2527. 
28. Pelliccia A, Di Paolo FM, Quattrini FM, Basso C, Culasso F, Popoli G, et al. Outcomes in athletes with marked ECG repolarization abnormalities. N Engl J Med 2008;358:152-161.

29. Maron BJ, Wolfson JK, Ciro E, Spirito P. Relation of electrocardiographic abnormalities and patterns of left ventricular hypertrophy identified by 2-dimensional echocardiography in patients with hypertrophic cardiomyopathy. Am J Cardiol 1983;51:189-194.

30. Rowin EJ, Maron BJ, Appelbaum E, Link MS, Gibson CM, Lesser JR, et al. Significance of false negative electrocardiograms in preparticipation screening of athletes for hypertrophic cardiomyopathy. Am J Cardiol 2012;110:1027-1032.

31. Riding NR, Sheikh N, Adamuz C, Watt V, Farooq A, Whyte GP, et al. Comparison of three current sets of electrocardiographic interpretation criteria for use in screening athletes. Heart 2014;101:384-390.

32. Kim JH, Baggish AL. Electrocardiographic right and left bundle branch block patterns in athletes: prevalence, pathology, and clinical significance. J Electrocardiol 2015;48:380-384.

33. Aro AL, Anttonen O, Tikkanen JT, Junttila MJ, Kerola T, Rissanen HA, et al. Intraventricular conduction delay in a standard 12-lead electrocardiogram as a predictor of mortality in the general population. Circ Arrhythm Electrophysiol 2011;4:704-710.

34. Surawicz B, Childers R, Deal BJ, Gettes LS, Bailey JJ, Gorgels A, et al. AHA/ACCF/ HRS recommendations for the standardization and interpretation of the electrocardiogram: part III: intraventricular conduction disturbances: a scientific statement from the American Heart Association Electrocardiography and Arrhythmias Committee, Council on Clinical Cardiology; the American College of Cardiology Foundation; and the Heart Rhythm Society: endorsed by the International Society for Computerized Electrocardiology. Circulation 2009; 119:e235-e240.

35. Marek J, Bufalino V, Davis J, Marek K, Gami A, Stephan W, et al. Feasibility and findings of large-scale electrocardiographic screening in young adults: data from 32,561 subjects. Heart Rhythm 2011;8:1555-1559.

36. Cohen MI, Triedman JK, Cannon BC, Davis AM, Drago F, Janousek J, et al. PACES/HRS expert consensus statement on the management of the asymptomatic young patient with a Wolff-ParkinsonWhite (WPW, ventricular preexcitation) electrocardiographic pattern: developed in partnership between the Pediatric and Congenital Electrophysiology Society (PACES) and the Heart Rhythm Society (HRS). Endorsed by the governing bodies of PACES, HRS, the American College of Cardiology Foundation (ACCF), the American Heart Association (AHA), the American Academy of Pediatrics (AAP), and the Canadian Heart Rhythm Society (CHRS). Heart Rhythm 2012;9:1006-1024.

37. Schwartz PJ, Stramba-Badiale M, Crotti L, Pedrazzini M, Besana A, et al. Prevalence of the congenital long-QT syndrome. Circulation 2009;120:1761-1767.

38. Meyer L, Stubbs B, Fahrenbruch C, Maeda C, Harmon K, Eisenberg M, et al. Incidence, causes, and survival trends from cardiovascular-related sudden cardiac arrest in children and young adults 0 to 35 years of age: a 30-year review. Circulation 2012;126:1363-1372.

39. Postema PG, De Jong JS, Van der Bilt IA, Wilde AA. Accurate electrocardiographic assessment of the QT interval: teach the tangent. Heart Rhythm 2008;5:1015-1018.

40. Bazett HC. An analysis of the time-relations of electrocardiograms. Heart 1920;353-370.

41. Malfatto G, Beria G, Sala S, Bonazzi O, Schwartz PJ. Quantitative analysis of T wave abnormalities and their prognostic implications in the idiopathic long QT syndrome. J Am Coll Cardiol 1994;23:296-301.

42. Ackerman MJ, Zipes DP, Kovacs RJ, Maron BJ. Eligibility and disqualification recommendations for competitive athletes with cardiovascular abnormalities: Task Force 10: The cardiac channelopathies: a scientific statement from the American Heart Association and American College of Cardiology. J Am Coll Cardiol 2015;66:2424-2428.

43. Goldenberg I, Moss AJ, Peterson DR, McNitt S, Zareba W, Andrews ML, et al. Risk factors for aborted cardiac arrest and sudden cardiac death in children with the congenital long-QT syndrome. Circulation 2008;117:2184-2191. 
44. Schwartz PJ, Moss AJ, Michael Vincent GM, Crampton RS. Diagnostic criteria for the long QT syndrome - an update. Circulation 1993;88: 782-784.

45. Bayes de Luna A, Brugada J, Baranchuk A, Borggrefe M, Breithardt G, Goldwasser D, et al. Current electrocardiographic criteria for diagnosis of Brugada pattern: a consensus report. J Electrocardiol 2012;45:433-442.

46. Biffi A, Pelliccia A, Verdile L, Fernando F, Spataro A, Caselli S, et al. Long-term clinical significance of frequent and complex ventricular tachyarrhythmias in trained athletes. J Am Coll Cardiol 2002;40:446-452.

47. Corrado D, Basso C, Leoni L, Tokajuk B, Turrini P, Bauce B, et al. Three dimensional electroanatomical voltage mapping and histologic evaluation of myocardial substrate in right ventricular outflow tract tachycardia. J Am Coll Cardiol 2008;51:731-739.

48. Gati S, Sheikh N, Ghani S, Zaidi A, Wilson M, Raju H, et al. Should axis deviation or atrial enlargement be categorised as abnormal in young athletes? The athlete's electrocardiogram: time for re-appraisal of markers of pathology. Eur Heart J 2013;34:3641-3648.

49. Kim JH, Noseworthy PA, McCarty D, Yared K, Weiner R, Wang F, et al. Significance of electrocardiographic right bundle branch block in trained athletes. Am J Cardiol 2011;107:1083-1089.

50. Finocchiaro G, Papadakis M, Robertus JL, Dhutia H, Steriotis AK, Tome M, et al. Etiology of Sudden Death in Sports: Insights From a United Kingdom Regional Registry. J Am Coll Cardiol. 2016 May 10;67(18):2108-2115. 